\title{
Twelve year outcomes following bacterial meningitis: further evidence for persisting effects
}

\author{
Keith Grimwood, Peter Anderson, Vicki Anderson, Lesley Tan, Terry Nolan
}

\begin{abstract}
Aim-To determine whether intellectual and cognitive impairments observed seven years following early childhood bacterial meningitis persist into adolescence. Methods-Blinded neuropsychological, auditory, and behaviour assessments were conducted in $109(69 \%)$ subjects from an original cohort of 158 children, seven and 12 years after their meningitis, and in 96 controls.

Results-Meningitis subjects remained at greater risk than controls for any disability (odds ratio OR 4.7 , confidence interval 2.2 to 9.6 ). Those with acute neurological complications had more sequelae than children with uncomplicated meningitis or controls $(47 \%$ v $30 \%$ v $11.5 \%$ respectively; $\mathbf{p}<0.001)$. Differences in intellectual, academic, and high level cognitive function between subjects and controls were maintained at the seven and 12 year assessments. In contrast, lower order skills improved, while behaviour scores deteriorated significantly $(p=0.033)$.

Conclusions-Many of the deficits identified at the seven year follow up persist 12 years after an episode of bacterial meningitis.
\end{abstract}

(Arch Dis Child 2000;83:111-116)

Keywords: meningitis; complications; developmental problems

Department of

Paediatrics, University

of Melbourne,

Melbourne, Australia

K Grimwood

T Nolan

Department of

Behavioural Science,

University of

Melbourne

P Anderson

V Anderson

Department of

Audiology, Royal

Children's Hospital,

Melbourne, Australia

L Tan

Correspondence to: Prof. K Grimwood, Department of Paediatrics and Child Health, Wellington School of Medicine, PO Box 7343, Wellington South,

New Zealand 6015

email:

grimwood@wnmeds.ac.nz

Accepted 15 February 2000

meningitis, ${ }^{2}$ responsible for over 200000 cases and more than 40000 deaths annually. ${ }^{23}$ Moreover, Neisseria meningitidis and Streptococcus pneumoniae remain important pathogens. ${ }^{4}$ Recurring epidemics of meningococcal disease, ${ }^{5}$ increased antibiotic resistance among pneumococci, ${ }^{6}$ and failure to introduce conjugate $\mathrm{Hib}$ vaccines into many developing countries means that bacterial meningitis remains a serious global health problem.

In spite of potent antibiotics and improved management of the critically ill, there is a small and significant risk of death or severe neurological sequelae following bacterial meningitis in childhood. A meta analysis found that $4.5 \%$ died and at least one major adverse outcome (severe intellectual disability, epilepsy, spasticity, deafness) was present in $16.4 \%$ of survivors. ${ }^{7}$ Inasmuch as these studies were lim- ited to neurological examinations and tests of general intellectual function or hearing acuity, with assessments restricted to one or two years follow up, the long term sequelae may have been underestimated. Many motor and cognitive skills are undeveloped at the time of meningitis. Consequently, functionally important deficits may not appear until the children are much older, attending school, and expected to think and reason independently.

Most meningitis survivors are considered to lead normal lives ${ }^{8}$ and to be little different from their siblings. ${ }^{9}$ Despite these impressions, a prospective seven year follow up study of children surviving bacterial meningitis and their classroom peers showed that these primary school age survivors showed mildly decreased intellectual quotient (IQ) scores and consistently performed less well with neuropsychological tasks, being more likely to have abnormal findings across all categories tested. ${ }^{10}$ The pattern of results suggested that their greatest impairment was in verbal skills and organisational capacity. ${ }^{11}$ Compared with $11 \%$ of controls exhibiting minor disabilities, $27 \%$ of children surviving meningitis had either neurological and behaviour disorders or cognitive impairments that may have contributed to their poorer academic performance. The risks for these adverse outcomes were greatest in those with meningitis during infancy and where there had been delays in diagnosis or acute neurological complications. ${ }^{11} 12$

The present study aimed to reassess the original cohort 12 years after their meningitis when many were early high school age. We determined whether previously observed disabilities persisted, suggesting permanent neurological deficits, if there was delayed acquisition of skills that improved with maturity, and if new deficits emerged with development.

\section{Methods}

SUBJECTS

A prospective cohort of 166 children, aged 3 months to 14 years, admitted to the Royal Children's Hospital, Melbourne with bacterial meningitis was established during October 1983 to September $1986 .{ }^{10}$ All were managed by a standardised protocol, which included penicillin and chloramphenicol as initial therapy. Overall, eight children died, leaving a cohort of 158 survivors.

Between 1991 and 1993, 130 (82\%) of the surviving cohort were evaluated at a mean age of 8.4 (SD 1.6) years and a mean of 6.7 (SD 0.8 , range 5.3-9.3) years since their meningitis. ${ }^{10-12}$ At the same time, grade and sex 
matched controls who had not suffered meningitis were recruited from each postmeningitis child's classroom by selecting the next same sex student on the class roll.

During 1996 and 1997, 109 (84\%) meningitis survivors who participated in the seven year follow up study were reassessed at a mean of 11.5 (SD 0.9, range 9.9-13.9) years after their meningitis. Additionally, $96(74 \%)$ of the original controls were also re-evaluated. No statistically significant differences were found in the demographic and clinical characteristics between the subjects in this study and those lost to follow up. Also when the adjusted IQ (WISC-R) scores at the seven year follow up for meningitis (98.7 (SE 1.4)) and control $\left(104.4\right.$ (SE 1.5)) ${ }^{10}$ children were compared with those not participating in the present study (97.2 (SE 4.1) v 103.0 (SE 2.0)), the relative difference between subjects and controls was maintained.

The mean ages of participating meningitis subjects and controls were 12.7 (SD 1.6, range $10-18$ ) and 13.0 (SD 1.7, range 10-18) years respectively. Sixty (55\%) subjects and 51 $(53 \%)$ controls were male. The maternal level of education (secondary $v$ postsecondary or tertiary) and the principal parental occupation score (Daniel Scale) ${ }^{13}$ determined socioeconomic status. Twenty nine per cent of mothers of children who had meningitis had a tertiary education compared with $35 \%$ of controls. The mean Daniel Score of Occupational Prestige was 4.4 (SD 1.3) for subjects and 4.1 (SD 1.1; $\mathrm{p}=0.047)$ for controls.

ETHICS

Approval for this study was obtained from the Royal Children's Hospital Ethics in Human Research Committee, and enrolment was by written informed consent from each child and their caregiver.

OUTCOME MEASURES

Evaluations were completed in fixed order during half day sessions by researchers unaware of the child's status. Psychological evaluation included overall intellectual ability, neuropsychological skills, academic achievement, central auditory function, and judgements by parents and teachers of each child's behaviour, school performance, and adaptive abilities. ${ }^{10}{ }^{14-19}$ Hearing for each ear was established by pure tone audiometry. The diagnostic threshold for deafness was a three frequency average at 500,1000, and $2000 \mathrm{~Hz}$ above 25 $\mathrm{dB}$ where hearing loss was defined as mildmoderate (25-69 dB) and severe-profound $(>70 \mathrm{~dB})$. Conductive deafness was differentiated from sensorineural losses by bone conduction testing.

\section{STATISTICAL ANALYSIS}

Data comparisons were performed using STATA. ${ }^{20}$ Bivariate, multivariate, and longitudinal analyses of nominal and continuous outcome variables were by $\chi^{2}$ tests, logistic regression, McNemar tests, two sample $t$ tests, multivariate analysis of covariance, and paired $t$ tests respectively. To determine changes over time for IQ and behaviour scales, the scores for each meningitis and control subject at the seven year assessment were subtracted from those obtained at their 12 year review. Ninety five per cent confidence intervals (CI) for proportions were by the exact binomial method. Multivariate analyses included gender, age at testing, maternal education, and parental level of occupation as covariates to adjust for the effects of age, gender, and social factors when comparing cases and controls.

\section{Results}

Table 1 summarises adverse outcomes; meningitis subjects were more likely than controls to have a disability. Nine (8.5\%; CI: 4 to 15$)$ had major neurological, auditory, or intellectual impairments. Another 32 (29.5\%; CI: 21 to 39) had less severe disabilities compared with 11 (11.5\%; CI: 6 to 20) controls, giving an attributable excess risk of $18 \%$ for minor

Table 1 Summary of impairments

\begin{tabular}{|c|c|c|c|c|c|c|}
\hline & \multicolumn{3}{|c|}{ Meningitis subjects } & \multicolumn{2}{|c|}{ Control subjects } & \multirow[b]{3}{*}{$95 \% C I$} \\
\hline & \multicolumn{3}{|c|}{ Complications* } & \multirow[b]{2}{*}{$(n=96)$} & \multirow[b]{2}{*}{$O R$} & \\
\hline & Yes $(n=49)$ & No $(n=60)$ & Total $(n=109)$ & & & \\
\hline Major impairment $\dagger$ & $7(14 \%)$ & $2(3 \%)$ & $9(8 \%)$ & 0 & & \\
\hline IQ $<70$ & $3(6 \%)$ & $1(2 \%)$ & $4(4 \%)$ & 0 & & \\
\hline Spasticity & $2(4 \%)$ & 0 & $2(2 \%)$ & 0 & & \\
\hline Blind & $1(2 \%)$ & 0 & $1(1 \%)$ & 0 & & \\
\hline Deaf $(\geqslant 70 \mathrm{~dB})$ & $3(6 \%)$ & 0 & $3(3 \%)$ & 0 & & \\
\hline Epilepsy & $3(6 \%)$ & $1(2 \%)$ & $4(4 \%)$ & 0 & & \\
\hline VP shunt & $2(4 \%)$ & 0 & $2(2 \%)$ & 0 & & \\
\hline Minor impairment $\neq$ & $16(33 \%)$ & $16(27 \%)$ & $32(29 \%)$ & $11(11 \%)$ & 3.2 & 1.5 to 6.7 \\
\hline IQ $70-80$ & $1(2 \%)$ & $4(7 \%)$ & $5(5 \%)$ & $3(3 \%)$ & 1.5 & 0.4 to 5.8 \\
\hline Educational deficits & $5(11 \%)$ & $6(10 \%)$ & $11(10 \%)$ & $3(3 \%)$ & 3.5 & 1.0 to 15.9 \\
\hline Deaf $(25-69 \mathrm{~dB})$ & $4(8 \%)$ & $3(5 \%)$ & $7(7 \%)$ & 0 & & \\
\hline Behaviour problems & $13(28 \%)$ & $12(20 \%)$ & $25(23 \%)$ & $7(7 \%)$ & 3.8 & 1.6 to 9.8 \\
\hline Summary & & & & & & \\
\hline No problems & $26(53 \%)$ & $42(70 \%)$ & $68(62 \%)$ & $85(89 \%)$ & 0.2 & 0.1 to 0.4 \\
\hline One minor impairment & $8(16 \%)$ & $8(13 \%)$ & $16(15 \%)$ & $6(6 \%)$ & 2.6 & 1.0 to 7.4 \\
\hline Significant impairment $\rrbracket$ & $15(31 \%)$ & $10(17 \%)$ & $25(23 \%)$ & $5(5 \%)$ & 5.4 & 2.0 to 14.3 \\
\hline
\end{tabular}

^Acute neurological complications = seizures, coma, VP shunt, hemiparesis, persistent hypotonia, visual loss, ataxia, and sensorineural deafness.

†Major $=\mathrm{IQ}<70$, spasticity, blind, severe-profound sensorineural deafness $(\geqslant 70 \mathrm{~dB})$, epilepsy, VP shunt.

$\ddagger$ Minor = IQ 70-80, educational deficit (reading, spelling, or maths SS $<70$ ), mild-moderate sensorineural deafness $(25-69 \mathrm{~dB})$, behaviour problem (CBCL or TRF summary $t$ score $>63) .{ }^{18} 19$

§Significant $=$ one major and/or more than one minor impairment. 
impairment. Overall, meningitis subjects were at substantially greater risk of an adverse outcome (odds ratio (OR) 4.7; CI: 2.2 to 10.0). Children with acute neurological complications had more sequelae than children with an uncomplicated illness ( $47 \%$ v 30\%; OR 2.1; CI: 0.93 to 4.6). Additionally, meningitis subjects without acute neurological complications remained at significantly greater risk of disability than controls (30\% v 11.5\%; OR 3.3; CI: 1.4 to 7.8 )

Table 2 shows the principal neuropsychological and behavioural findings. As a group, meningitis subjects again showed significantly lower IQ scores and poorer academic abilities. While the mean adjusted Full Scale IQ for the meningitis subjects was within the normal range, it was significantly lower than controls (97.1 (range 48-136) v 101.6 (range 78-135) respectively). In the previous four years, 29 $(27 \%)$ subjects and $12(12.5 \%)$ controls received educational assistance (OR 2.5; CI: 1.2 to 5.5 ), which was significantly greater than reported at the seven year review $(5.5 \% v 0 \%$ respectively; $\mathrm{p}<0.001$ ).

Table 2 also shows that meningitis subjects performed more poorly than controls in a number of neuropsychological domains. Unlike the seven year assessment, there were no significant group differences for lower order, routine abilities such as attention, speeded response, and immediate memory capacity.
However, subjects showed consistently lower scores on tasks requiring high level skills including complex linguistic ability, new learning, and executive functions such as organisation, problem solving, and mental flexibility.

Child Behaviour Checklists (CBCL) were completed for 198 (97\%) meningitis subjects and controls, while 179 (87\%) Teacher's Report Forms were returned from the schools. Higher scores indicated a greater likelihood of clinical psychiatric problems. ${ }^{18}{ }^{19}$ Subjects were on average rated to have more behaviour problems, especially for internalising behaviours of somatic complaints, mood, social, thought and attention problems, and delinquent behaviour.

Figure 1 illustrates the mean IQ and CBCL behaviour scores for both meningitis subjects and controls at the seven and 12 year assessments, while table 3 shows the mean differences in change scores during these times. Overall, the differences in intellectual function between subjects and controls persisted. In contrast, the changes in mean behaviour scores were significantly greater in controls than meningitis subjects, especially for internalising behaviours. The greater fall in scores shown by controls suggested fewer behaviour problems. Comparing the rates of CBCL total behaviour scores within the clinical range supported this proposition. While at the seven and 12 year assessments, the proportion of meningitis subjects with CBCL total behaviour scores above

Table 2 Principal neuropsychological and behavioural findings

\begin{tabular}{|c|c|c|c|c|c|}
\hline & \multicolumn{2}{|l|}{ Adjusted means * } & \multirow[b]{2}{*}{ Mean difference } & \multirow[b]{2}{*}{$95 \% C I$} & \multirow[b]{2}{*}{$p$ value } \\
\hline & $\begin{array}{l}\text { Meningitis }(n=107) \\
\text { Mean }(S E)\end{array}$ & $\begin{array}{l}\text { Control }(n=96) \\
\text { Mean }(S E)\end{array}$ & & & \\
\hline \multicolumn{6}{|l|}{ WISC-III ${ }^{14} \dagger$} \\
\hline Verbal IQ & $95.8(1.2)$ & $100.0(1.2)$ & 4.2 & 0.9 to 7.5 & 0.014 \\
\hline Performance IQ & $99.2(1.3)$ & $103.4(1.3)$ & 4.2 & 0.6 to 7.8 & 0.023 \\
\hline Full scale IQ & $97.1(1.1)$ & $101.6(1.2)$ & 4.5 & 1.2 to 7.8 & 0.007 \\
\hline \multicolumn{6}{|l|}{ Academic achievement ${ }^{15}$} \\
\hline WRAT-3 Reading & $99.0(1.3)$ & $104.2(1.4)$ & 5.3 & 1.4 to 9.1 & 0.008 \\
\hline WRAT-3 Spelling & $95.5(1.3)$ & $101.4(1.3)$ & 5.9 & 2.3 to 9.6 & 0.002 \\
\hline WRAT-3 Mathematics & $94.6(1.1)$ & $97.8(1.2)$ & 3.2 & 0.0 to 6.3 & 0.048 \\
\hline \multicolumn{6}{|l|}{ Language $^{16}$} \\
\hline Making inferences & $26.6(0.5)$ & $28.8(0.5)$ & 2.2 & 0.8 to 3.6 & 0.002 \\
\hline Oral expression & $13.7(0.4)$ & $14.3(0.4)$ & 0.6 & -0.4 to 1.6 & 0.253 \\
\hline \multicolumn{6}{|l|}{ Memory and new learning ${ }^{15}$} \\
\hline Max digit span - auditory memory & $5.8(0.1)$ & $5.9(0.1)$ & 0.1 & -0.2 to 0.4 & 0.576 \\
\hline RAVLT - verbal learning & $51.5(0.7)$ & $53.5(0.8)$ & 2.0 & -0.1 to 4.0 & 0.060 \\
\hline RVDLT_-visual learning & $41.2(0.9)$ & $45.8(0.9)$ & 4.6 & 2.0 to 7.2 & 0.001 \\
\hline $\mathrm{RCF}$ —delayed recall & $19.4(0.5)$ & $21.2(0.6)$ & 1.8 & 0.2 to 3.4 & 0.025 \\
\hline \multicolumn{6}{|l|}{ Attention and processing speed ${ }^{17}$} \\
\hline Code test - focused and sustained attention & $37.1(0.3)$ & $37.7(0.3)$ & 0.6 & -0.2 to 1.5 & 0.136 \\
\hline Sky search-processing speed & $77.5(1.8)$ & $72.9(1.9)$ & -4.6 & -9.9 to 0.7 & 0.088 \\
\hline \multicolumn{6}{|l|}{ Executive functions ${ }^{15}$} \\
\hline $\mathrm{RCF}$ - accuracy & $31.7(0.3)$ & $33.1(0.3)$ & 1.4 & 0.5 to 2.3 & 0.004 \\
\hline $\mathrm{RCF}$-organisation & $4.7(0.1)$ & $5.1(0.1)$ & 0.4 & 0.1 to 0.7 & 0.004 \\
\hline Tower of London-planning & $4.5(0.2)$ & $4.0(0.2)$ & -0.6 & -1.0 to -0.1 & 0.012 \\
\hline COWAT - verbal generation & $25.8(0.8)$ & $28.1(0.9)$ & 2.3 & -0.1 to 4.6 & 0.060 \\
\hline \multicolumn{6}{|l|}{ Behavioural and social adjustment } \\
\hline \multicolumn{6}{|l|}{ CBCL $(\mathrm{T} \text { scores })^{18}$} \\
\hline School competence & $45.6(0.8)$ & $48.8(0.8)$ & 3.2 & 0.9 to 5.3 & 0.005 \\
\hline Internalising & $51.1(1.2)$ & $46.5(1.3)$ & -4.6 & -8.1 to -1.2 & 0.008 \\
\hline Externalising & $50.6(1.0)$ & $47.0(1.1)$ & -3.6 & -6.5 to -0.7 & 0.015 \\
\hline Total problems & $51.3(1.1)$ & $46.2(1.2)$ & -5.1 & -8.3 to -1.9 & 0.002 \\
\hline \multicolumn{6}{|l|}{ TRF $(\mathrm{T} \text { scores })^{19} \neq$} \\
\hline Internalising & $48.8(0.9)$ & $47.6(1.0)$ & -1.2 & -3.8 to 1.4 & 0.366 \\
\hline Externalising & $48.8(0.9)$ & $48.2(1.0)$ & -0.6 & -3.2 to 2.1 & 0.658 \\
\hline Total problems & $48.5(0.9)$ & 46.9 (1.0) & -1.6 & -4.3 to 1.1 & 0.248 \\
\hline
\end{tabular}

*Adjusted for gender, age, and sociodemographic variables at the 12 year follow up assessment.

+WISC-III, Weschler Intelligence Scale for Children, 3rd ed.; WRAT-3, Wide Range Achievement Test, 3rd ed.; RAVLT, Rey Auditory Verbal Learning Test; RVDLT, Rey Visual Design Learning Test; RCF, Rey Complex Figure; COWAT, Controlled Oral Word Association Test; CBCL, Child Behaviour Checklist; TRF, Teacher's Report Form.

$\ddagger$ Completed TRF questionnaires were returned by schools for 94 children who had meningitis and for 85 controls. 

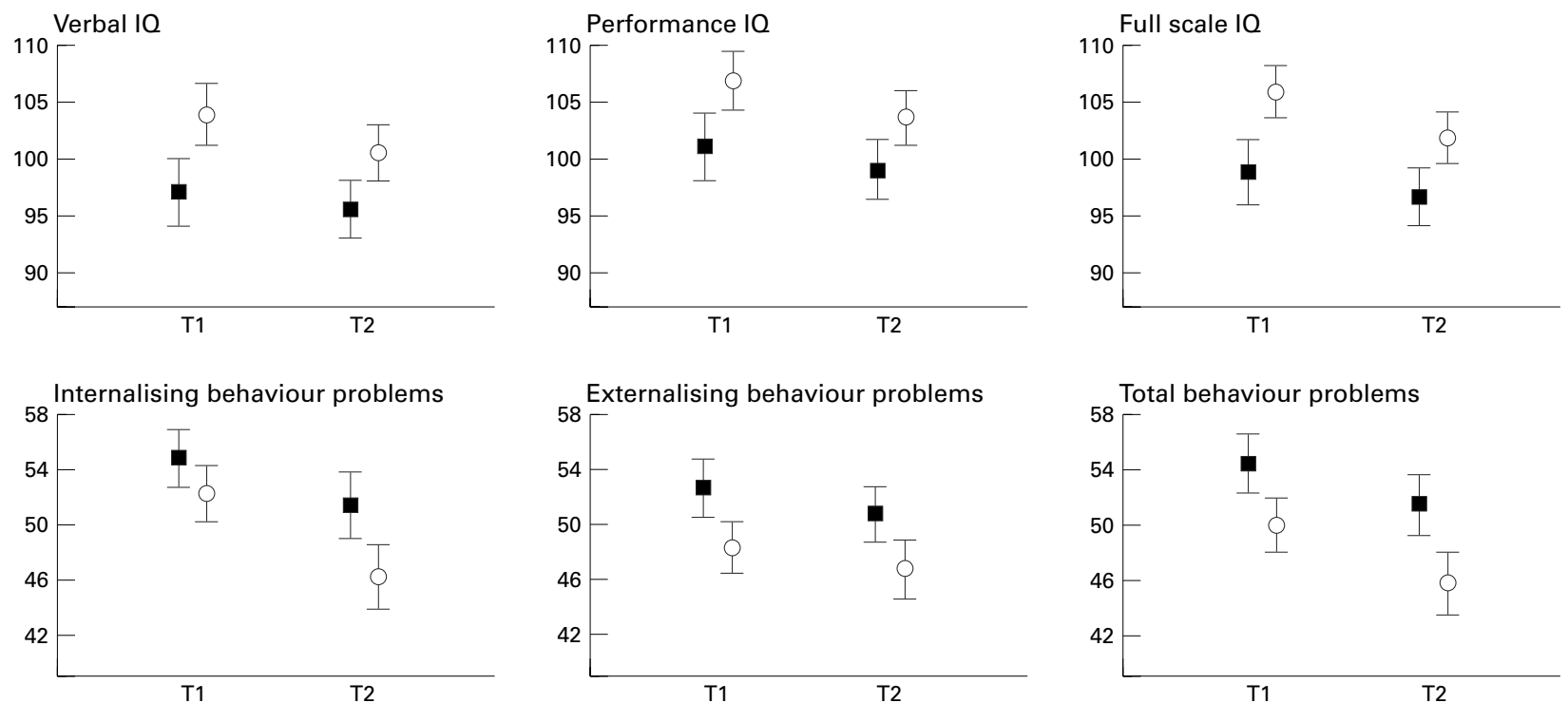

Meningitis subjects

Control subjects

Figure 1 Mean (95\% CI) for intellectual quotient (IQ) and Child Behaviour Checklist (CBCL) behaviour problem scores from meningitis and control subjects at seven (T1) and 12 year (T2) assessments. Scores were adjusted for gender, age, and sociodemographic variables at the time of assessment. Changing from the WISC-R to WISC III instrument at the T1 and T2 assessments respectively accounts for as much as five points difference in IQ scores. ${ }^{14}$ Higher $C B C L$ scores are associated with a greater risk of clinical psychiatric problems. ${ }^{18}$

63 was similar at $23 \%$ and $18 \%$ respectively, within controls the corresponding rates had decreased significantly from $11 \%$ to $4 \%$ $(\mathrm{p}=0.02)$.

Ten meningitis subjects had sensorineural deafness (9.2\%; CI: 4.5 to 16.2), including nine with losses of the same type and degree recorded at the seven year assessment. ${ }^{10}$ One meningitis subject had developed a bilateral mild mid frequency loss since the earlier assessment and was subsequently fitted with hearing aids. Tests of central auditory function were completed in 99 meningitis and 86 control subjects. They were not conducted in those with hearing aids or if visits were out of working hours. Compared with controls, there was an increased risk for abnormal responses among subjects in tests of short term auditory memory (STAM) for sentences $(21.2 \% v$ $7.0 \%$; OR 3.6; CI: 1.3 to 10.0 ) and auditory figure ground speech discrimination (for example, $25 \%$ v $7.0 \%$ at $+5 \mathrm{db}$; OR 4.8 ; CI: 1.8 to 12.5). For respective seven and 12 year assessments there was little change in the proportion of subjects $(17 \% v 21 \%)$ and controls $(7 \% v$

Table 3 Intellectual quotient and behaviour mean difference scores between seven (T1) and 12 year (T2) assessments

\begin{tabular}{lllll}
\hline & $\begin{array}{l}\text { Meningitis subjects } \\
\text { adjusted means }(S E)\end{array}$ & $\begin{array}{l}\text { Control subjects } \\
\text { adjusted means }(S E) *\end{array}$ & $\begin{array}{l}\text { 95\% CI difference in } \\
\text { change scores (T2-T1) }\end{array}$ & p value \\
\hline WISC-IIIt & & & -1.7 to 3.1 & 0.558 \\
VIQ & $-3.2(0.8)$ & $-2.5(0.9)$ & -1.3 to 4.0 & 0.323 \\
PIQ & $-3.7(0.9)$ & $-2.4(1.0)$ & -1.9 to 2.4 & 0.835 \\
FSIQ & $-3.5(0.7)$ & $-3.3(0.8)$ & -6.3 to -0.5 & 0.021 \\
CBCL $\neq$ & & $-6.4(1.1)$ & -4.3 to 0.7 & 0.161 \\
Internalising & $-3.0(1.0)$ & $-2.7(0.9)$ & -5.6 to -0.2 & 0.033 \\
Externalising & $-0.9(0.9)$ & $-5.1(1.0)$ & \\
Total problems & $-2.2(0.9)$ & &
\end{tabular}

*Adjusted for T1 score, gender, age at T2, SES at T2, and time since T1.

+WISC-R at T1; WISC-III at T2 (change in tests meant that WISC IQ scores were as much as five points lower). ${ }^{14}$

$\ddagger$ Child Behaviour Checklist where higher scores indicate a greater likelihood of clinical psychiatric problems. ${ }^{18}$
$7 \%$ ) with abnormal STAM responses for sentences. In contrast, the respective rates of abnormal responses for tests of auditory figure ground speech discrimination between the two assessments for subjects $(60 \%$ v $25 \%$; $\mathrm{p}<0.001)$ and controls $(37 \%$ v $7 \%$, $\mathrm{p}<0.001)$ had reduced significantly.

\section{Discussion}

Children assessed seven and 12 years after meningitis remain at significant risk for neurological and auditory abnormalities. Compared with controls they functioned at significantly lower levels for measurements of intelligence and high level neuropsychological skills and had more behavioural difficulties at home and at school. The risk for sequelae was greatest in those who experienced acute neurological complications at the time of their illness. However, there was no evidence of further intellectual or cognitive deterioration. Instead some lower order skills, such as attention, processing speed, and immediate memory capacity displayed improvement from below age level to normal at the seven and 12 year assessments respectively. High level cognitive skills including organisational skill, problem solving, verbal fluency, and mental flexibility continue to be impaired at the 12 year assessment. This may reflect an ongoing "lag" in development with executive functions still maturing in our sample. ${ }^{112122}$ Central auditory function also improved, although difficulties with language based tasks remain. In contrast, while most sensorineural hearing loss following meningitis remains stable, spontaneous fluctuations or even progression may occur more than 12 years after recovery. ${ }^{23} 24$

The difficulty is that it is not currently possible to predict whether children who had meningitis will continue to develop these skills. 
While this is suggested by our serial data, others have postulated that development of higher order skills remains incomplete, ${ }^{25}$ although such findings are based on subjects with residual brain pathology, in contrast to meningitis survivors where the cerebral insult is often transient. There are limited data in children undergoing serial neuropsychological assessments after bacterial meningitis. Twenty four Hib meningitis survivors and their matched sibling pairs were examined seven and 11 years later for educational outcomes. ${ }^{26}{ }^{27}$ Although minor differences in IQ scores and neuropsychological function between them persisted, academic achievements for meningitis children and their sibling controls remained comparable. However, retrospective recruitment from medical records and restricting subjects to those with near age siblings increases the risk of non-representative sampling, and sibling controls may be adversely affected by the extra care and attention received by the index child. ${ }^{28}$

The children in this cohort are broadly representative of the paediatric meningitis population from an industrialised country before introduction of conjugate $\mathrm{Hib}$ vaccines. ${ }^{10}$ The sociodemographic and clinical characteristics of subjects and those lost to follow up were comparable. Furthermore, the $14.5 \%$ prevalence of severe neurological sequelae, including hearing loss, is similar to that of other prospective studies in unselected patients. ${ }^{7}$ Except for small differences in parental occupation, meningitis and control subjects remained alike for other sociodemographic factors. Similar differences in the seven year mean IQ scores between subjects and controls participating in the 12 year assessments and in those lost to follow up further suggests that retention bias is unlikely.

Longitudinal research in children surviving meningitis is restricted by the psychometric limitations of the tests, age based variations in standard scores, and necessary changes in test protocols. The slightly lower IQ scores recorded for both groups at the 12 year review may be accounted for by the different tests employed at the seven and 12 year assessments (WISC-R and WISC-III respectively). Data available comparing these tests suggest that the WISC-III will record a full scale IQ score approximately five points lower than the WISC-R. ${ }^{14}$ Similarly, our controls showed decreased (that is improved) CBCL scores over time, consistent with observations from population based studies. ${ }^{29}$ The meningitis group did not follow this trend, possibly indicating a relative increase in behavioural problems associated with academic difficulties and low self esteem.

Since the cohort's inception, conjugate Hib vaccines have virtually eliminated this pathogen from many industrialised countries. ${ }^{14}$ However, wherever the vaccine is unavailable, $\mathrm{Hib}$ meningitis is a public health problem. ${ }^{23}$ Furthermore, penicillin and chloramphenicol without dexamethasone is still the initial treatment for bacterial meningitis in developing countries. ${ }^{30}$ As meningococci and pneumococci are also important causes of meningitis,

\section{Key messages}

- Bacterial meningitis in children is associated with substantial excess risk of intellectual, cognitive, and auditory impairment that persists into adolescence

- Continuing developmental problems of higher order language, organisation, problem solving, and central auditory function may increase learning and behavioural difficulties

- The risk of these adverse outcomes is greatest in, but not confined to, those who experienced acute neurological complications at the time of their illness

- Families, schoolteachers, and health professionals have an important role in identifying and/or helping those with learning and behavioural difficulties

large numbers of bacterial meningitis survivors will continue to be at moderate risk of mild developmental problems directly attributable to their illness, with associated learning and behaviour difficulties at school and continuing into adulthood. ${ }^{11}{ }^{24}$

As children without identifiable risk factors may still develop functionally important disabilities following meningitis, ${ }^{12}$ families and schoolteachers should be made aware of possible language deficits and problems comprehending language based material. Auditory figure ground problems compound these difficulties, and in a noisy classroom children may not hear instructions and may not always understand what they do hear. Early learning programmes that include quieter classrooms, sitting close to the teacher, small group teaching, repetition of information, rephrasing verbal material, and practice may help compensate for these learning deficits, resulting in improved academic achievement, behaviour, and self esteem. ${ }^{27}$

This study was financed by grants from the Government Employee's Medical Research Fund and the Royal Children's Hospital Research Institute. We thank J Pollard for assistance with audiological testing, $\mathrm{S}$ Vadmir for assistance with fig 1 , and the members of the families who agreed to participate in this study.

1 Robbins JB, Schneerson R, Anderson P, Smith DH. Prevention of systemic infections, especially meningitis, caused by Haemophilus influenzae type b. Impact on public health and implications for other polysaccharide-based vaccines. IAMA 1996;276:1181-5.

2 Salisbury DM. Summary statement: The first international Salisbury DM. Summary statement: The first international
conference on Haemophilus influenzae type $\mathrm{b}$ infection in conference on Haemophilus influenzae type
Asia. Pediatr Infect Dis $\mathcal{F} 1998 ; 17: S 93-5$.

3 Mulholland K, Hilton S, Adegbola R, et al. Randomised trial of Haemophilus influenzae type-b tetanus protein conjugate for prevention of pneumonia and meningitis in Gambian infants. Lancet 1997;349:1191-7.

4 Schuchat A, Robinson K, Wenger JD, et al. Bacterial meningitis in the United States in 1995. N Engl f Med 1997;337: $970-6$.

5 Booy R, Kroll JS. Bacterial meningitis and meningococcal infection. Curr Opin Pediatr 1998;10:13-18.

6 Goldstein FW, Acar JF, and The Alexander Project Collaborative Group. Antimicrobial resistance among lower respiratory tract isolates of Streptococcus per isolates of Streptococcus USA collaborative surveillance study. $\mathcal{F}$ Antimicrob Chemother 1996;38: (suppl A)71-4.

7 Baraff LJ, Lee SI, Schriger DL. Outcomes of bacterial meningitis in children: a meta-analysis. Pediatr Infect Dis $\mathcal{f}$ 1993;12:389-94. 
8 Davies PA. Long-term effects of meningitis. Dev Med Child Neurol 1989:31:398-406.

9 Taylor HG, Mills EL, Ciampi A, et al. The sequelae of Haemophilus influenzae meningitis in school-age children. $N$ Engl f Med 1990;323:1657-63.

10 Grimwood K, Anderson VA, Bond L, et al. Adverse outcomes of bacterial meningitis in school-age survivors. Pediatrics 1995;95:646-56.

11 Anderson VA, Bond L, Catroppa C, Grimwood K, Keir E, Nolan T. Childhood bacterial meningitis: impact of age at illness and acute medical complications on long term outcome. FINS 1997;3:147-58.

12 Grimwood K, Nolan TM, Bond L, Anderson VA, Catroppa C, Keir E. Risk factors for adverse outcomes of bacterial meningitis. F Paediatr Child Health 1996;32:457-62.

13 Daniel A. Power, privilege and prestige. Occupations in Australia. Melbourne: Longman Cheshire, 1983.

14 Wechsler D. Manual for the Wechsler Scale of Children's Intelligence-III. New York: Psychological Corporation, Intelligen

15 Lezak M. Neuropsychological assessment. New York: Oxford, 1993.

16 Wiig E, Secord H. Test of language competence: expanded edition. San Antonio, TX: Psychological Corporation, 1989.

17 Manly I, Robertson I, Anderson V. Test of everyday attention for children. Sussex, UK: Thames Valley Test Company, 1999.

18 Achenbach TM. Manual for the Child Behavior Checklist 4-18 and 1991 profile. Burlington, VT: University of Vermont Department of Psychiatry. 1991

19 Achenbach TM. Manual for the Teacher's Report Form and 1991 profile. Burlington, VT: University of Vermont Department of Psychiatry, 1991.

20 StataCorp. Stata Statistical Software: Release 5.0. College Station, TX: Stata Corporation, 1997.
21 Levin H, Culhane K, Hartmann J, et al. Developmental changes in performance on tests of purported frontal lobe

22 Anderson V. Assessing executive functions in children: biological, psychological and developmental considerations. Neuropsychological Rehabilitation 1998;8:319-49.

23 Silkes ED, Chabot ED. Progressive hearing loss following Haemophilus influenzae meningitis. Int $\mathcal{F}$ Pediatr Otorhinolaryngol 1985;9:249-56.

24 Hugosson S, Carlsson E, Borg E, Brorson L-O, Langeroth G, Olcen P. Audiovestibular and neuropsychological outcome of adults who had recovered from childhood bacterial meningitis. Int $\mathcal{F}$ Pediatr Otorrhinolaryngol 1997;42: 149-67.

25 Dennis M. Language and the young damaged brain. In: Boll T, Bryant B, eds. Clinical neuropsychology and brain function. Research, measurement and practice. Washington: American Psychological Association, 1989:85-124.

26 Taylor HG, Michaels RH, Mazur PM, et al. Intellectual, neuropsychological, and achievement outcomes in children six to eight years after recovery from Haemophilus influensix to eight years after recovery from Haemophi
zae meningitis. Pediatrics 1984;74:198-205.

27 Feldman HM, Michaels RH. Academic achievement in children ten to 12 years after Haemophilus influenzae meningitis

28 D'Angio CT, Froehlke RG, Plank GA, et al. Long-term outcome of Haemophilus influenzae meningitis in Navajo Indian children. Arch Pediatr Adolesc Med 1995;149:10018.

29 Verhulst FC, Koot HM, Berden GFMG. Four-year follow-up of an epidemiological sample. I Am Acad Child Adolesc Psychiatry 1990;29:440-8.

30 Qazi SA, Khan MA, Mughal N, et al. Dexamethasone and bacterial meningitis in Pakistan. Arch Dis Child 1996;75: $482-8$.

\section{STAMPS IN PAEDIATRICS}

\section{Child welfare}

The only stamp to promote and publicise child helplines comes from Denmark. This was issued in 1991 for the 25th anniversary of Living Conditions of Children, a child welfare organisation. A child using the emergency helpline is depicted and the stamp carries a surcharge for the charity.

M K DAVIES

A J MAYNE

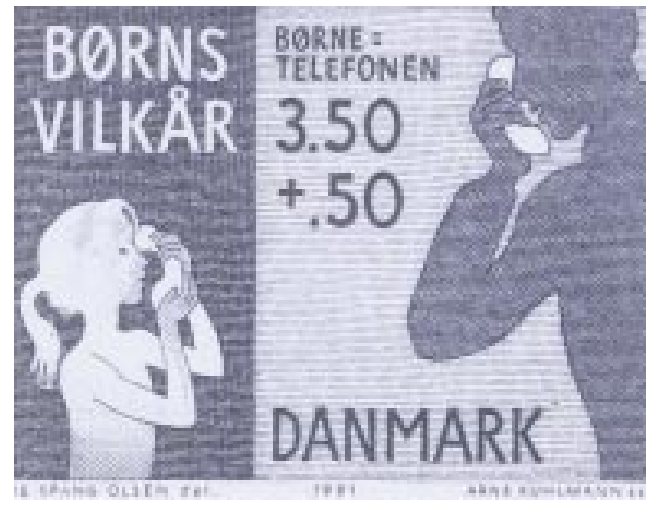

